\title{
DE LA POLÍTICA DEL SUJETO AL SUJETO POLÍTICO. ${ }^{1}$
}

\author{
FROM POLITICS OF THE SUBJECT TO THE \\ POLITICAL SUBJECT: \\ EDUCATION FOR EMANCIPATION
}

Gina Marcela Arias Rodríguez. ${ }^{2}$

Fabián F. Villota Galeano. ${ }^{3}$

\begin{abstract}
Palabras Clave: Sujeto Político, público-privado, transformación, educación formal-no formal, emancipación, resistencia, crítica.

Key words: Political subject, emancipation, public-private, transformation, formal and non-formal education, resistance, critique.
\end{abstract}

\section{RESUMEN.}

En este artículo se abordan las concepciones tradicionales del sujeto político para ampliarlas a través de una caracterización del juego dialéctico entre las dimensiones pública y privada en las que se desenvuelve, la noción emanada de esta discusión es confrontada con algunas condiciones del conflicto colombiano. Interpelando así la noción tradicional de sujeto político; asumiéndolo de manera compleja, como un sujeto en constante construcción a partir de estrategias y mecanismos que le permitan disponerse a la realidad, y mejorar y transformar la suya propia. En este sentido, se valora el proceso educativo (formal y no formal) que pueda potenciar la formación de dicho sujeto hacia la emancipación.

\section{ABSTRACT.}

This article approaches the traditional conceptions about the political subject in order to widen them through a characterization of a dialectical game between the public and the private dimensions in which he acts.
1 El Presente artículo se elabora a partir de las reflexiones hechas en la Línea de Investigación en Psicología Social: Conflicto y Violencia de la Universidad Católica Popular del Risaralda y se basa en la ponencia presentada en el marco de la IV Jornada de Psicología. Universidad Católica Popular del Risaralda. Octubre de 2006.

2 Psicóloga, UCPR. DocenteInvestigadora Universidad Católica Popular del Risaralda. Estudiante de Maestría en Psicología Comunitaria, Universidad de Chile. Santiago de Chile.

3 Antropólogo, Universidad de Caldas. DocenteInvestigador Universidad Católica Popular del Risaralda. Estudiante de Maestría en Antropología Social, Universidad de Antioquia. Medellín.

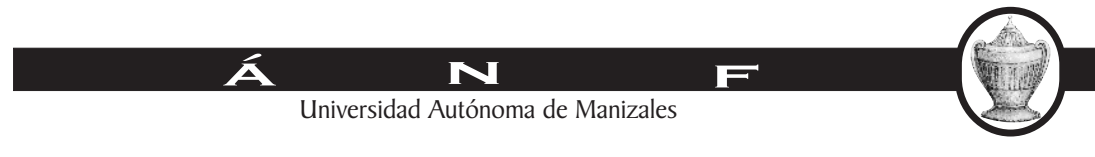


The notion resulting from this discussion is confronted with the context of the Colombian conflict. It is assumed, from this perspective, that the Political Subject is not a finished subject, but it is a subject in permanent construction, who is constantly looking for strategies and mechanisms to improve and transform his own reality. Accordingly, the educational process (both formal and non-formal) may potentiate the subject's structuring.

\section{I. ¿Por qué de la Política del Sujeto al Sujeto Político?}

El título que da nombre a este artículo implica una consideración inicial: La "Política del sujeto" puede ser asumida en una doble acepción. La primera: como una serie de principios estructurados y coherentes de los que dispone el sujeto para orientar sus prácticas en las que se ponen de manifiesto un juego de intereses y/o de poderes. La segunda acepción se asumiría como una estructura objetiva que dispone al sujeto en un juego de intereses y/o poderes.

En el caso del sujeto político la cuestión aparece un poco más clara, el sujeto político es el sujeto imbuido de la condición política; lo político aquí es una cualidad, una condición que define la naturaleza del sujeto.

Mas allá de la inversión de los términos, entre la primera cuestión y la segunda aparece una diferencia: por un lado parece que una cosa es la política y otra es lo político, lejos de que nuestra intención sea sumarnos a la distinción propuesta por A. Heller (1991), quisiéramos que se entendiese concretamente que una cosa es la política como un sistema que da coherencia a unos tipos de prácticas (las que ponen en juego intereses, o un juego de poderes) y otra lo político como una cualidad que caracteriza ciertas prácticas (las que ponen en juego intereses y poderes, por ejemplo).

En las dos acepciones de la "política del sujeto", la política aparece como fija, de hecho de aquí deviene su carácter estructural (esto no quiere decir que no pueda ser modificada). Por el contrario no puede decirse esto de la condición que define la naturaleza del sujeto del que hablamos aquí, lo político en el sujeto no es fijo, el carácter de su condición es ser procesual, es decir que está en permanente construcción. 
Esta condición procesual es lo que da la posibilidad a la reflexión sobre la permanente construcción y formación del sujeto Político. Es en este sentido, donde se plantea que el proceso educativo (formal y no formal) tiene que ver mucho con esa posibilidad e intencionalidad de contribuir en la formación de sujetos autónomos, responsables, solidarios, críticos, reflexivos.

\section{EI Sujeto Político: la tensión entre lo público y lo privado.}

Retomemos ahora las características que tradicionalmente han definido al sujeto político:

- Se interesa por trascender del ámbito individual al colectivo; del ámbito privado al público. Hay preeminencia de lo público sobre lo íntimo, de lo colectivo sobre lo individual.

- Asume una actitud reflexiva sobre su condición de ser político.

- Se asume como constructor de su propia realidad.

- Reconoce la responsabilidad que tiene frente a la necesidad de transformar la realidad.

- Esa transformación la logra mediante la acción organizada y reflexionada.

Preguntémonos si ces posible considerar al sujeto instalado exclusivamente en una sola dimensión: la pública (que es la dimensión donde oficia como tal)? Esto con el fin de interpelar la idea de que el sujeto político se hace al pasar de la dimensión privada a la pública, y proponer la idea de que el sujeto político se construye en una suerte de trayectoria primero y de simultaneidad después entre lo público y lo privado y que le resulta coherente.

Al pensar que el sujeto político se construye en una trayectoria y en una simultaneidad, asumimos su constitución como dinámica entre las dimensiones, y no exclusivamente instalada en una u otra dimensión. Sin embargo la primacía de la dimensión pública sobre la privada está en entre dicho, para esto pensemos en la forma en que se asume una regla o una norma: generalmente hay obediencia a la regla cuando se presupone que así el sujeto se beneficia; de la misma manera, la norma se viola cuando se considera "más" benéfico la violación que la obediencia (Bourdieu, 2002). Creemos que una relación de este tipo opera para el concepto de sujeto político, él no abandona su dimensión privada, ni "sacrifica" su intimidad por el interés público, lo que sucede 
es que logra que haya una coherencia en los intereses que tiene como individuo y los intereses que tiene como agente (portador de capitales públicos). Como en el caso de la norma, sólo en la medida en que el interés público, por el que se supone el sujeto político toma partido, no suponga la abolición de los intereses privados, se convierte en competencia para el individuo.

En la idea de la primacía de la dimensión pública sobre la privada subyace otra cuestión, la de que el interés del sujeto político está desprovisto de intencionalidades para sí, y no se trata de plantear el asunto en términos de valores como el altruismo y el egoísmo sino de la manera en que éste logra coherencia entre lo que considera de interés para sí y los intereses públicos.

Asumiendo así la naturaleza del concepto, despojamos del halo "mesiánico" al sujeto político: no es literalmente un sujeto gregario que se debe exclusivamente al colectivo, antes bien su individualidad adquiere sentido como correlato de los intereses del colectivo, de alguna manera se sintetiza así: los intereses para sí son correspondientes con los intereses públicos o colectivos (esto no quiere decir que sean los mismos, sino que son de la misma naturaleza); es decir, su ánimo público redunda en favor de su dimensión privada, es ahí donde él también gana. Otro asunto es la consideración de los capitales que considera gananciales a la hora de establecer balances y comparaciones con otros agentes.

Al asumir una actitud reflexiva sobre su condición de ser político; esto es cuando reflexiona sobre su interés y el de los demás, lo que se impone no es uno de los intereses, lo que se impone es la coherencia y correspondencia entre unos y otros.

Otra característica del Sujeto Político tiene que ver con la idea de que se asume como constructor de su propia realidad, en este caso, lo que presupone la afirmación es que su existencia no está supeditada a lo que la realidad social le plantea para sí; es decir, él no es un cumplidor de lo que está establecido, él asume que la realidad es una construcción social y el papel que cumple no es el de ser consecuencia de la estructuración social sino que se siente parte de ella, de ahí su disposición a creer que la puede transformar a través de su acción y su 
reflexión porque no delega el orden social en otros, sino que se hace responsable de ese orden. De aquí deviene la idea de que el sujeto político es un sujeto histórico, lo es porque la historia la hacen los hombres y no la estructura que se supone los ordena y dispone.

Si como hemos dicho al principio, lo político es una condición, en este caso una condición del sujeto a través de la cual guía ciertas prácticas que ponen en juego intereses y poderes, aquí la naturaleza de esa condición estaría determinada por todas las características que hemos acotado hasta aquí. Pero nos parece que lo político es algo más que una simple condición con cierta naturaleza; con el fin de pensar más ampliamente lo político recurrimos a una idea de la antropología, de la antropología política específicamente, y de un autor en particular; Marc Abeles (1997) quien delimita lo político como:

Una forma elaborada de juego, en el cual están implicadas dos tipos de regla: las reglas normativas que son definidas como reglas del juego oficial aceptadas por los adversarios, y las reglas pragmáticas que ponen en obra las estrategias concurrentes. Estos análisis ponen de relieve la noción de toma de decisión. ${ }^{4}$

Asumido en esta dimensión de lo político, el sujeto político es el jugador, el que se dispone coherentemente entre las reglas normativas y las reglas pragmáticas; es decir, entre las reglas que le permiten reconocer en qué ámbito se está moviendo y las reglas que le permiten distinguir sus movimientos como autónomos. Al reconocer los tipos de regla reconoce cómo operan en cada ámbito de su existencia, y así el sujeto encuentra la coherencia entre los intereses para sí y los intereses públicos o colectivos. Trasciende en la medida en que puede reconocerlos, no en la medida en que sacrifica los unos a los otros, trasciende en la medida en que reconoce cómo establecer las relaciones para capitalizar el juego de intereses; por decirlo de alguna manera, el sujeto político juega siempre a ganar, no en el sentido de la apuesta, sino en la medida en que equilibra la capitalización a corto plazo / en el ámbito individual con la capitalización a largo plazo / en el ámbito colectivo o público, entre lo que es coyuntural y lo que es estructural.

Cuando se cree que el sujeto político está dispuesto hacia el bien común, es cuando se asume como un individuo sacrificial, casi
4 Abélès, M. (1997), "El lugar de la política". EN: Revista Internacional de Ciencias. Sociales No 153.

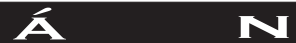

Universidad Autónoma de Manizales

\section{$\mathbf{R}$}

Año I4, Número 23, julio - diciembre 2007 
mesiánico, que prefiere subordinar el interés privado al público, y no es de esta manera, porque si bien su acción puede estar orientada hacia el bien común, este no lo excluye, él hace parte de ese "común", inclusive él instituye los indicadores de lo que es el común, él es el autorizado para "dictar" los lineamientos que delimiten el asunto.

Pero ¿Qué o quién es entonces un sujeto político? No es un individuo dotado de virtudes que le endilga la sociedad sobre la que resulta relevante, a fuerza de su accionar. Ante todo el sujeto político es un estratega, un buen jugador, para usar las acepciones de Abeles, justamente porque su apariencia es la de ser "uno de los mismos, como nosotros... pero destacado", estratega porque sabe disponerse de manera capitalizable entre sus intereses y los del colectivo que lo hacen posible; porque dispone de una estrategia para no reducir sus intereses a los de los demás ni viceversa, hay correspondencia entre lo que pretende el colectivo y las pretensiones de él como individuo, pero como él es el mediador, puede hacerlo de manera tal que concilia el ámbito privado y el público, sin lograr dependencia el uno del otro. Su legitimidad está en la ascendencia que tiene sobre el colectivo.

Así entonces la condición de político del sujeto se da cuando reconoce cuál es el juego entre dos tipos de reglas y que se resuelven en una suerte de formalización, por ejemplo: la ciudadanía. Asumamos por un momento que el sujeto político emerge a través de esta categoría: ser ciudadano, más allá de las acepciones filosóficas con la que se presenta, supone la aceptación y disposición a una serie de reglas que garantizan la pertenencia o no a dicha categoría, de ahí que la idea de ciudadanía este supeditada a la idea de sujeto de deberes y de derechos.

¿Cuál es el tipo de sujeto político que se está modelando a partir de lo dicho hasta aquí? Hemos desprovisto al Sujeto Político de su carácter mesiánico, para instituirlo como un sujeto que equilibra los intereses fundados en los ámbitos privados y públicos; por ejemplo, al mantener la idea de que su emergencia se formaliza a través de la ciudadanía, pareciese que se está proponiendo un sujeto "cumplidor de la ley". Para el caso de lo establecido en la constitución Colombiana, la ciudadanía se garantiza a través de posibilitar el ejercicio de la participación; en ese sentido, los ciudadanos no son sólo sujetos depositarios de los beneficios del modelo de Estado, es decir, su relación no es

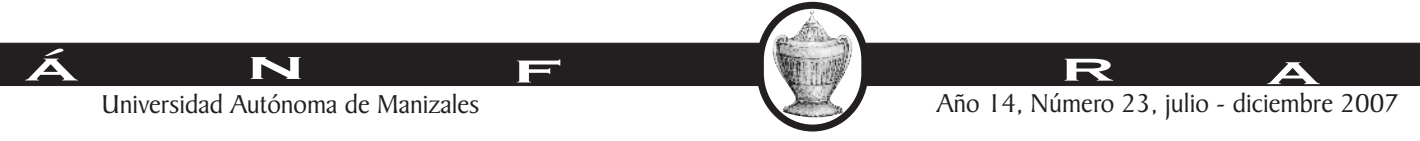


exclusivamente pasiva, sino que al ser ciudadanos o al reivindicarse como tales, deben ser activos deben ser sujetos de deberes.

Así entonces, el sujeto político deseado por las concepciones elaborados hasta aquí, puede ser el ciudadano; y es así por la acepción de que lo político también está asociado a la acción dirigida hacia el colectivo, donde los individuos que se erigen como sujetos políticos ejercen liderazgo, responsabilidad, control social, etc. y se convierten en veedores de los deberes y derechos sociales. ¿Deberíamos quedarnos con esta conceptualización acerca del sujeto político?

Es aquí donde hace carrera la idea de que las categorías que elabora el científico social, luego de pretender abarcar la realidad, pretenden producir el advenimiento de ésta (Bourdieu, 1997). El sujeto político que se evidencia hasta aquí es el sujeto político que se desea y que subyace en la acotación misma del concepto.

Esta es otra razón por la cual asumimos una idea mucho más amplia, y también delimitada de lo político. Creemos que cuando se asume la idea de lo político como la de una forma elaborada de juego, en el cual están implicados unas reglas, no se está estableciendo un juicio acerca del tipo de juego que debe jugar el sujeto político manifiesto en esta condición; es decir, el sujeto político no necesariamente es quien juega el "buen juego" (así éste sea un juego elaborado) sino que es el que reconoce el tipo de reglas en el que está inmerso y en medio de las cuales sabe moverse, porque sabe que son las que lo hacen posible.

En ese sentido, la emergencia del sujeto político no se hace manifiesta exclusivamente a través de la ciudadanía; es decir, no sólo es sujeto político el individuo que ejerce la ciudadanía (la de los derechos y los deberes), porque ésta implica además del reconocimiento de las reglas del juego político el apego a "una" de las formas de la regla: la legal, la jurídica. Porque si suponemos que el sometimiento a la regla sólo se da cuando se gana más que violándola, quien opere así en el ámbito político está reconociendo el juego al que se ve avocado.

Suponemos que algo similar ocurre con los sujetos políticos, que ese sujeto deseable que busca el concepto elaborado anteriormente: el ciudadano respetuoso de las normas, pero veedor de los derechos y deberes, etc., es una de las formas de este sujeto, pero que hay otras,

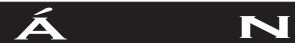

Universidad Autónoma de Manizales 
que tiene incidencia en ese juego complejo que es lo político: piénsese en el funcionario corrupto, y en nuestro contexto en los miembros de los grupos de autodefensa o de los grupos guerrilleros que plantean o a quienes se les plantean negociaciones, rebaja de penas, indultos, etc., con tal de resolver su posición en el juego, donde la característica que los distingue de los demás es que no es legal, pero la consideran legítima; es sobre esa premisa que descansa la posibilidad de "negociar" con ellos.

Todo esto para hablar de otros sujetos políticos que inciden en ese juego de reglas y que pretenden el logro de objetivos, etc. Pero de los que no puede decirse que no pretenden el bien común, ni mucho menos que no reflexionan sobre el contexto social y político en el que se desenvuelven para buscar estrategias de resolución (aunque es bien conocida en muchas ocasiones las propuestas acerca del Estado, de la sociedad, de la economía, etc. que vienen de todos los bandos: guerrilla, autodefensas, etc.). Estos nuevos sujetos emergentes también son políticos y no por las valoraciones que se hacen sobre su accionar, sino porque están en ese juego complejo. Es una suerte de sujeto político no deseado, del que no pretendemos su advenimiento ni su producción, pero que en el caso nuestro existe y sobre el cual las representaciones que hacemos conceptualmente, deben dar cuenta.

Superada la idea del sujeto político que simpatiza con los valores políticos que se desean de un líder: el bienestar general, la primacía del interés colectivo sobre el interés particular, y asentada en la idea de que se trata de un estratega transparente que sabe cómo conciliar sus intereses con los de los demás, que gana haciendo que ganen todos; pasamos a la idea del sujeto que se pliega al otro lado del asunto, el sujeto que también es un estratega, pero que no es manifestación de esos principios políticos deseables, por el contrario él justifica su violación a la regla reconociéndolo no como método legal, pero sí legítimo; y reconociendo a su vez, de alguna manera, el tipo de regla normativa que lo hace posible: piénsese en los grupos armados cuando no reconocen las leyes del Estado colombiano pero a su vez pretenden negociar con él, considerándolo interlocutor válido. Definitivamente hay un reconocimiento de la regla normativa, por eso pueden "negociar"; pero les distingue la norma pragmática: ellos son "beligerantes, sediciosos, etc."

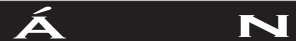

Universidad Autónoma de Manizales 
¿Puede negarse a este tipo de agentes la categoría de sujeto político? no serán el sujeto político deseado pero de hecho hacen parte activa de ese juego complejo, que nos hemos dado en llamar político y que actualmente vive la nación.

¿Puede creerse en Colombia de la irrelevancia política de los grupos armados ilegales? otra cosa es que esa relevancia sea deseable o no, pero que existe, existe. Tanto que muchas leyes y muchas de las acciones políticas del Estado están orientadas considerando el accionar de estos grupos: piénsese en la ley de sometimiento a la justicia, en la ley de justicia y paz, en los acuerdos de paz o de los intercambios humanitarios.

Un asunto como estos puede considerarse irrelevante para alguien, pero eso no puede ser su política (una estructura), pero si alguien lo considera es indudable el carácter político de esa consideración.

\section{La Educación para la emancipación.}

Además de todas las características abordadas anteriormente, consideramos pertinente trabajar una más: la idea de que el sujeto político es un estratega que sabe articular coherentemente lo público y lo privado y también tiene una intencionalidad que es la de transformar el mundo que lo circunda y la realidad de la cual ha sido partícipe en su construcción. De esta manera, el sujeto político se asume como perteneciente al grupo, a la comunidad que le confiere sentido y de igual manera, asume que la realidad social no esta dada en sí misma por naturaleza, ni por la responsabilidad de los Otros Poderosos ${ }^{5}$ como el destino, la suerte, la gracia divina, etc. Se asume entonces que la realidad es socialmente construida y por ende susceptible de ser transformada, modificada por la acción misma de los sujetos, grupos o comunidades.

Lo anterior, invita a pensar en sujetos que son reflexivos, capaces de desarrollar una conciencia moral, una sensibilidad por el otro y por lo tanto, la posibilidad de la transformación no la asume solamente él (recuérdese que anteriormente se planteó la necesidad de despojar del halo mesiánico al sujeto político), sino que por el contrario se piensa que ésta -la transformación- es posible gracias al colectivo que ha hecho posible a ese sujeto político.
5 Montero, M. (2002) Introducción a la Psicología Comunitaria. Editorial Paidós. Buenos Aires.

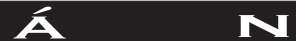

Universidad Autónoma de Manizales 
Cuando se plantea la posibilidad de la transformación no se puede dejar de lado el principio de Emancipación. Emancipar significa liberar: permitir que los sujetos salgan de situaciones complejas que los apabulla, los oprime. Un sujeto que se emancipa es un sujeto que cuestiona y pone en tela de juicio las cosas "tal y como son"; es decir, avanza en la desnaturalización de los fenómenos y las problemáticas propias de su contexto. ${ }^{6}$ Esta idea de la emancipación es la que propone Kant como proceso que se opone a la "minoría de edad"; es decir, que la emancipación es la capacidad que tiene el sujeto para la realización de sus acciones sin la dirección de otro, y éste no es más que el principio de autonomía. $^{7}$

La idea de una educación para la emancipación tiene mucho que ver con la formación de esos sujetos políticos y es aquí donde el científico social plantea la posibilidad de no sólo abarcar la realidad con las categorías que construye sino que también procura producir el advenimiento de ésta. Se produce entonces la siguiente pregunta: ¿Qué y cómo hacer para facilitar y promover un proceso de formación de sujetos políticos? En este sentido, se expresa la necesidad de apelar al proceso educativo (formal y no formal) como escenario que debería posibilitar la reflexión, la crítica, el cuestionamiento, la problematización de las cosas (de los hechos y los por hacer según Ignacio Martín-Baró) que se presentan en el contexto.

El proceso educativo entonces no es entendido solamente como la mera transmisión de datos y conocimientos sino que constituye en sí mismo la socialización, como plantea Rosa, A. (2004)

La educación puede ser considerada como un proceso de dos caras: por una parte, es una actividad social, para socializar y enculturar a los individuos que se han de incorporar en el grupo social (...) y por otra, es un proceso de entramamiento al que los individuos deben someterse para alcanzar destrezas que les permitan mejorar sus condiciones de integración social futuras. $^{8}$

De acuerdo a lo anterior, el sujeto que nos interesa formar como sujeto político es aquel que logra no sólo incorporarse al grupo social sino que también lo reflexiona, lo cuestiona y le interesa transformarlo para bien de sí mismo y del grupo al que pertenece. Además se entiende que en la
6 Notas del Seminario de Profundización en Psicología Socio-Educativa orientado por el Doctor en Psicología Social, Amalio Blanco Abarca.

7 Concepto que ha sido ampliamente desarrollado en la Psicología y que supone la estructuración misma del sujeto.

8 Rosa, A. (2004), "Enfoques Socioculturales y Educación" Universidad Autónoma de Madrid.

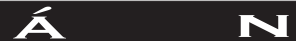

Universidad Autónoma de Manizales

\section{$\mathbf{R}$}

Año I4, Número 23, julio - diciembre 2007 
medida que se puedan alcanzar saberes, habilidades y destrezas se podrán abonar mejores condiciones de vida. De esta manera, asumimos que el espacio educativo es un escenario de socialización donde los sujetos encuentran maneras de organizarse, de participar, de discutir, de construir con el otro; es decir, es el escenario propicio para la formación de la capacidad crítica, democrática y participativa.

Pero también entendemos que el proceso educativo como tal, también se convierte en un espacio para reproducir y perpetuar prácticas que impiden la transformación para el bien individual y colectivo; por lo tanto, se expresa la necesidad de repensar y replantear algunas prácticas educativas que en cierta medida impiden abonar el terreno para la formación de sujetos políticos.

De acuerdo a lo anterior, apelamos a las premisas fundamentales de Adorno (1998) en Educación para la Emancipación.

1. Abandonar la vieja creencia romántica de la existencia de genios. Se asume que las capacidades de los individuos no son innatas sino que se construyen en el colectivo que lo hace posible y esto tiene estrecha relación con la idea que mantenemos de despojar del halo mesiánico al sujeto político. Dichas capacidades son posibles en el ámbito escolar, en el grupo, en lo comunitario. Para Adorno, "la capacidad es, en una proporción muy importante, función de las condiciones sociales, de tal modo que ya los propios presupuestos de la emancipación, de la que depende una sociedad libre, vienen determinados por la falta de libertad en la sociedad." ${ }^{\prime 9}$

2. La segunda idea que sostiene Adorno y que queremos resaltar de su discusión referente a la razón absoluta es que "sólo mediante el pensamiento se puede realizar algo así como una determinación de lo que es justo y adecuado hacer, de una praxis correcta." ${ }^{10}$ Es decir, que la reflexión, la crítica, el discernimiento que se supone debe promover la educación debe facilitar también un análisis de lo que es justo o no en el contexto, en el grupo, en la comunidad, en la sociedad y de esta manera derivar una praxis que permita introducir la posibilidad del cambio y la transformación.
9 Adorno, T. (1998), "Educación para la emancipación". Editorial Morata. Madrid.

10 Op. Cit.
A N

Universidad Autónoma de Manizales 
3. La tercera idea que se quiere resaltar es la que se refiere al fenómeno psicosocial de la autoridad y cómo este contribuye en la estructuración de un sujeto autónomo; por lo tanto, emancipado. Adorno hace alusión a las investigaciones que desarrolló Else Frenkel-Brunswik, donde se demuestra que son los niños que logran interiorizar las normas y los valores los que más adelante se constituyen en personas más autónomas y capaces de ejercer la oposición contra aquello que no consideran justo, más que aquellos niños refractarios, que se oponen y recitan discursos de oposición. Con esto, Adorno pretende manifestar que el fenómeno de la Personalidad Autoritaria ampliamente estudiado abordado por él no debe ser considerado igual a la relación de autoridad (no autoritarismo) que se debe construir en los diferentes escenarios de socialización: familia, escuela, grupos de pares, etc. Por lo tanto, la relación de autoridad que construya el sujeto con el maestro es el espacio educativo permitirá no sólo internalizar las normas y valores de manera acrítica sino reflexiva. Se aboga entonces por una relación de respeto por el otro, menos vertical y más horizontal.

4. Una educación para la emancipación pretende poner en tela de juicio aquello que las personas se tragan sin oponer ninguna resistencia, aquello que es transmitido y que se asume que así es y así debería ser por alguna razón que no necesariamente es conocida y reflexionada. $Y$ esto tiene relación con la posibilidad que propone Adorno de un yo consistente o débil. Un yo débil no cuestiona ni pregunta, se asume entonces que para dar lugar al advenimiento del sujeto político, se esperaría que éste vaya constituyendo un yo consistente que no necesariamente se adapte a las condiciones que el contexto le impone, sino que por el contrario pueda asimilar dichas condiciones y busque transformarlas. Una educación para la emancipación debe pretender entonces procurar una educación que también es política, que permita pasar de condiciones heterónomas a posturas más autónomas; es decir, a un yo cada vez más consistente. Lo anterior, permite pensar entonces que la emancipación no es un estado ideal, acabado, terminado, no es 
simple retórica. Emancipar, cambiar, transformar lleva implícita la palabra proceso. El proceso implica la formación y la participación permanente de los sujetos.

5. Finalmente, la Educación para la Emancipación es una educación para la contradicción y la resistencia ante lo que se impone. Es una educación que pretende que los sujetos tengan la posibilidad de develar, desenmascarar lo que se ha transmitido y reproducido a través de la mentira y el engaño. En este sentido, Adorno aboga por una educación que desacredita este tipo de prácticas. Pero también puede darse que los sujetos en su proceso de formación política tengan cierto sentimiento de impotencia ante lo que se considera es imposible de cambiar, al respecto Adorno (1998) expone "es posible que quien quiera transformar sólo pueda hacerlo en la medida en que convierta esta misma impotencia, junto con su propia impotencia, en un momento de lo que piensa y quizá también de lo que hace." ${ }^{11}$ Es decir, que el sentimiento de impotencia experimentado por los sujetos políticos sea asumido como un momento del proceso, pero entendiendo que es posible salir de ahí con la acción reflexionada y organizada.

\section{REFERENCIAS.}

ABÉLÈS, M. (1997), "El lugar de la política". EN: En: Revista Internacional de Cs. Sociales $n^{\circ} 153$.

ADORNO, T. (1998), "Educación para la emancipación". Editorial Morata. Madrid.

BOURDIEU, P. (1997), "Razones Prácticas". Editorial Anagrama. Barcelona.

BOURDIEU, P. (2002), "El Sentido Práctico". Editorial Taurus. Madrid.

HELLER, A. (1991) "¿Historia y futuro. Sobrevivirá la modernidad?" Barcelona: Península.

MONTERO, M. (2002) "Introducción a la Psicología Comunitaria". Editorial Paidos. Buenos Aires. 
ROSA, A. (2004), "Enfoques Socioculturales y Educación" Universidad Autónoma de Madrid.

\section{BIBLIOGAFIA COMPLEMENTARIA}

ARENDT, H. (1997). ¿Qué es la Política? Barcelona: Editorial Piados.

BOURDIEU, P; Passeron, J. (2003), "Los herederos, los estudiantes y la cultura". Siglo XXI editores. México D.F.

DÍAZ, Álvaro. (2003) "Una discreta diferenciación entre la política y lo político y su incidencia sobre la educación en cuanto socialización política". Manizales.

MONTERO, M. (1999) "De la Realidad, la Verdad y otras Ilusiones Concretas: Para una Epistemología de la Psicología Social Comunitaria". Revista PSYKHE. 1999. Vol. 8. No. 1, 9-17.

SEAONE. (1988) "Psicología Política". Editorial Pirámide. Madrid. 